\title{
Fréchet distance on the set of compact trees
}

\author{
Olha Lozinska, Aleksandr Savchenko, Mykhailo Zarichnyi
}

Abstract We introduce a counterpart of the Fréchet distance for the rooted trees in a metric space. Some properties and possible generalizations of this distance are discussed.

Keywords tree, Fréchet distance

Mathematics Subject Classification (2010) 54F50, 54B20, 37E25

UDK 515.12

\section{Introduction}

The Fréchet distance between two curves in a metric space was defined more than century ago. Informally, this distance is described as the minimal length of a leash that allows for a dog to walk along one curve and the owner along the other one.

There is a huge amount of literature devoted to the Fréchet distance. This distance has numerous applications in mathematics and computer science as well as in mathematical morphology. There are different versions of this distance, in particular, a nonmonotone (weak) (see, e.g., [1,2]), geodesic [7], homotopy [6], discrete etc. Fréchet distance.

One can also try to define counterparts of the Fréchet distance for another types of graphs. In particular, this is already done for circles (simple closed curves; [2]). It is worth noting that one can apply the Fréchet distance for circles in order to impose topology in the set of rosettes (locally convex closed curves $[5])$. 
In this note, we define a counterpart of the Fréchet distance for the trees. One of our motivations lies in possible applications to investigations of planar domains by means of their skeletons. Recall that the skeleton of a domain is the set of centers of the maximal balls in this domain. If the domain is sufficiently regular (e.g., is twice differentiable), then its skeleton is a tree that preserves (in some sense) the shape of this domain.

\section{Fréchet distance}

Given a topological space $X$, we say that a subspace $T$ of $X$ is a tree in $X$ if $T$ is homeomorphic to a (topological) tree.

A tree $T$ is rooted if a point base point (root) $t_{0} \in T$ is specified. We restrict ourselves with the compact rooted trees. We assume that all the maps between rooted trees are root-preserving.

Recall that a map of two topological spaces is monotone if the preimage of every point is connected.

A branch of a tree $T$ is a maximal (with respect to inclusion) embedded segment in a tree such that the root is one of its endpoints.

A map of trees is said to be branch-monotone if its restriction onto every branch is a monotone map. A norm on a rooted tree $T$ (with root $t_{0}$ ) is a continuous function $t \mapsto\|t\|: T \rightarrow \mathbb{R}_{+}$such that $\left\|t_{0}\right\|=0$ and which is an embedding on every branch of $T$.

Definition 1 Let $(X, d)$ be a metric space and let $T, S \subset X$ be rooted trees.

Define

$$
\begin{aligned}
D_{F}(T, S)= & \inf \{\sup \{d(\alpha(x), \beta(x)) \mid x \in R\} \mid \alpha: R \rightarrow T, \beta: R \rightarrow S \\
& \text { are monotone onto maps of a tree } R\} .
\end{aligned}
$$

We first remark that the function $D_{F}$ is well-defined. Indeed, consider the bouquet $T \vee S$ of $T$ and $S$, i.e., the space $(T \sqcup S) / \sim$, where $\sim$ identifies the roots in $T$ and $S$. There exist natural retractions $T \vee S$ onto $T$ and $S$. These retractions (sending, respectively, $S$ and $T$ to the root) are monotone onto maps.

Let $d_{H}$ denote the Hausdorff metric. Recall that

$$
d_{H}(A, B)=\inf \left\{r>0 \mid A \subset O_{r}(B), B \subset O_{r}(A)\right\}
$$

(by $O_{t}(Y)$ the $t$-neighborhood of a set $Y$ is denoted).

Lemma 1 The following inequality holds: $D_{F} \geq d_{H}$. 
Proof Suppose that $D_{F}(T, S)<c$, where $c>0$. There is a rooted tree $R$ and monotone onto maps $\alpha: R \rightarrow T$ and $\beta: R \rightarrow S$ such that $\sup \{d(\alpha(r), \beta(r)) \mid$ $r \in R\}<c$. Given $t \in T$, find $r \in R$ such that $\alpha(r)=t$. Then $d(t, \beta(r))<c$. Therefore, $T \subset O_{c}(S)$. Similarly, $S \subset O_{c}(T)$ and therefore $d_{H}(T, S) \leq c$.

Theorem 1 The function $D_{F}$ is a metric on the set of all trees in a metric space $(X, d)$.

Proof Clearly, $D_{F} \geq 0$. If $D_{F}(T, S)=0$, then, by Lemma $1, d_{H}(T, S)=0$ and thus $T=S$.

It is clear that the function $d_{F}$ is symmetric.

We are going to prove the triangle inequality for $D_{F}$. Let $T, S, R$ be rooted trees in $X, D_{F}(T, S)<a, D_{F}(S, R)<b$. Then there exist rooted trees $U, V$ and monotone onto maps $\alpha: U \rightarrow T, \beta: U \rightarrow S, \gamma: V \rightarrow S, \delta: V \rightarrow R$ such that, for every $u \in U$ and every $v \in V$, we have $d(\alpha(u), \beta(u))<a, d(\gamma(v), \delta(v))<b$.

Approximating, if necessary, the maps $\alpha, \beta, \gamma, \delta$ by maps that are embeddings when restricted to the branches one may assume, without loss of generality, that the maps $\alpha, \beta, \gamma, \delta$ themselves satisfy this property. (If necessary, one should attach short enough edges to the roots of $T, S, R$ and move the roots to the other ends of these edges. Note also that, if necessary, segments can be attached to the metric space $X$ under consideration, in order to make these changes of roots possible.)

Let $W$ be the pullback of the maps $U \rightarrow R \leftarrow V$. More precisely,

$$
W=\{(u, v) \in U \times V \mid \beta(u)=\gamma(v)\} .
$$

Define $\alpha^{\prime}: W \rightarrow T$ and $\delta^{\prime}: W \rightarrow R$ as follows: $\alpha^{\prime}(u, v)=\alpha(u), \delta^{\prime}(u, v)=\delta(v)$.

We are going to show that $W$ is a tree. Show that $W$ is contractible. Let $\|\cdot\|$ be a norm on $S$. First note that the map $(u, v) \mapsto \beta(u)=\gamma(v): W \rightarrow S$ induces a norm on $W$; we keep the notation $\|\cdot\|$ for this induced norm.

Now if $(u, v) \in W$, then there is segments $J, K$ in $U$ and $V$ respectively that connect $\left(u_{0}, v_{0}\right)$ to $(u, v)$. For every $\theta \in[0, \beta(u)]$ there are unique $u_{\theta} \in J$ and $v_{\theta} \in K$ such that $\left\|u_{\theta}\right\|=\left\|v_{\theta}\right\|=\theta$. Clearly, $\theta \mapsto\left(u_{\theta}, v_{\theta}\right)$ is a segment in $W$ that connects $\left(u_{0}, v_{0}\right)$ and $(u, v)$.

Define a map $H: W \times[0,1] \rightarrow W$ as follows. Let $H((u, v), \tau)$ be the point $\left(u_{\tau\|u\|}, v_{\tau\|u\|}\right)$, which is described above. Clearly, $H$ is a homotopy between the constant map with the value $\left(u_{0}, v_{0}\right)$ and the identity map $1_{W}$. We conclude that $W$ is a tree. 
Then

$$
\begin{aligned}
D_{F}(T, R) & \leq \sup \left\{d\left(\alpha^{\prime}(u, v), \delta^{\prime}(u, v)\right) \mid(u, v) \in W\right\} \\
& \leq \sup \{d(\alpha(u), \beta(u))+d(\gamma(v), \delta(v)) \mid(u, v) \in W\} \leq a+b
\end{aligned}
$$

which completes the proof.

Example 1 Let

$$
T=\{(x, 0) \mid-1 \leq x \leq 0\} \subset \mathbb{R}^{2}, S=\{(x, 0) \mid 0 \leq x \leq 1\} \subset \mathbb{R}^{2},
$$

with the origin as the root of both $T$ and $S$. Denote the Fréchet distance by $\delta_{F}$. It is easy to demonstrate that $\delta_{F}(T, S)=2$.

In order to show that $D_{F}(T, S)=1$, let $W=T \cup S$. We assume that the origin is the root of $W$. Let $\alpha: W \rightarrow T, \beta: W \rightarrow S$ be branch-monotone retractions. Then $D_{F}(T, S) \leq \sup \{d(\alpha(w), \beta(w)) \mid w \in W\}=1$. Since $D_{F} \geq d_{H}$, we are done.

Let $\left(C, c_{0}\right)$ be a curve with chosen endpoint $c_{0}$ (root). For any point $c \in C$, denote by $c^{-}$the part of $C$ between $c_{0}$ and $c$, by $c^{+}$we denote the closure of the complement of $c^{-}$.

Proposition 1 Let $T, S$ be curves whose roots are endpoints. Then, for every $t \in T, s \in S$, we have

$$
D_{F}(T, S) \leq \max \left\{\delta_{F}\left(t^{-}, s^{-}\right), d_{H}\left(t^{+},\{s\}\right), d_{H}\left(\{t\}, s^{+}\right)\right\}
$$

Proof Let $U$ be a triode, i.e., a graph which is a bouquet of three segments. Let $u, v, w$ be three points of degree 1 in $U$. Let $c$ be the point of degree 3 in $U$. We suppose that $u$ is the root of $U$. Now, define $\alpha:[u, c] \rightarrow t^{-}, \beta:[u, c] \rightarrow s^{-}$be maps such that the Fréchet distance between $t^{-}$and $s^{-}$is reached.

Next, define $\alpha^{\prime}: U \rightarrow T$ by the conditions: $\alpha^{\prime}\left|[u, c]=\alpha, \alpha^{\prime}\right|[c, v]$ is an arbitrary monotone map onto $t^{+}, \alpha^{\prime} \mid[c, w]$ is a constant map taking the value $s$. Similarly, define $\beta^{\prime}: U \rightarrow S$ by the conditions: $\beta^{\prime}\left|[u, c]=\beta, \beta^{\prime}\right|[c, v]$ is a constant map taking the value $t$, and $\beta^{\prime} \mid[c, w]$ is an arbitrary monotone map onto $s^{+}$. Then $\sup \{d(\alpha(x), \beta(x)) \mid x \in U\}$ is exactly the right-hand side of (1) and this completes the proof.

We do not have, however, any estimate of $D_{F}$ from below (excepting $d_{H}$ ). 


\section{Remarks and open questions}

One can also define a notion of Fréchet distance for non-rooted trees; namely, let

$$
\tilde{D}_{F}(T, S)=\inf \left\{D_{F}\left(\left(T, t_{0}\right),\left(S, s_{0}\right) \mid t_{0} \in T, s_{0} \in S\right)\right\} .
$$

Simple examples demonstrate that $\tilde{D}_{F} \neq D_{F}$.

A dendrite is a locally connected (metrizable) continuum that contains no simple closed curves [4]. It seems plausible that our definition of distance between rooted trees can be easily modified into an analogous definition of distance between dendrites in a metric space. It is an open question whether the space of trees would be dense in the space of dendrites.

H. Alt and M. Godau [2] investigated computational properties of the Fréchet distance. In particular, they showed that there exists an algorithm that computes the distance between two polygonal curves in time $O(m n \log m n)$, where $m, n$ are numbers of vertices of the curves. We formulate the problem of finding algorithms for computing the distance between polygonal trees. Note also that for computational purposes it would be convenient to define a discrete version of the Fréchet distance between trees in the spirit of [8].

\section{References}

1. H. Alt and M. Godau. Measuring the resemblance of polygonal curves. In: Proc. 8th Annu. ACM Sympos. Comput. Geom., pages 102-109, 1992.

2. H. Alt and M. Godau. Computing the Fréchet distance between two polygonal curves. Internat. J. Comput. Geom. Appl., 5:75-91, 1995.

3. L.E. Bazylevych, M.M. Zarichnyi, On metrization of the hyperspace of oriented arcs, Visnyk Lviv. univ. Ser. Mech.-Math. 1996. Volume 43, P. 3-5. (in Ukrainian)

4. Janusz J. Charatonik, and Wlodzimierz J. Charatonik, Dendrites, Aportaciones Matemáticas, Serie Comunicaciones 22 (1998) 227-253.

5. W. Cieślak, W. Mozgawa, On rosettes and almost rosettes, Geometriae Dedicata, October 1987, Volume 24, Issue 2, pp 221-228.

6. Atlas F. Cook IV and Carola Wenk. Geodesic Fréchet distance inside a simple polygon. In: Proc. 25th Symp. Theoretical Aspects of Computer Science (STACS), 193-204, 2008.

7. Alon Efrat, Leonidas J. Guibas, Sariel Har-Peled, Joseph S. B. Mitchell, and T. M. Murali. New similarity measures between polylines with applications to morphing and polygon sweeping. Discrete Comput. Geom., 28:535-569, 2002.

8. T. Eiter and H. Mannila, Computing discrete Fréchet distance, Technical Report CD-TR 94/64, Christian Doppler Laboratory for Expert Systems, TU Vienna, Austria, 1994.

\section{Olha Lozinska}

Department of Mechanics and Mathematics, Lviv National University, Universytetska Str. 1, 79000 Lviv, Ukraine

E-mail: olja.Iviv133@gmail.com 


\section{Aleksandr Savchenko}

Kherson State Agrarian University, 23 Rozy Liuksemburg Str., 73006 Kherson, Ukraine

E-mail: savchenko1960@rambler.ru

\section{Mykhailo Zarichnyi}

Department of Mechanics and Mathematics, Lviv National University, Universytetska Str. 1, 79000 Lviv, Ukraine

E-mail: mzar@litech.lviv.ua 